Piero LEO* - Gianpaolo RuZZANTE**

\title{
Dati nuovi e riassuntivi sui Tenebrionidi dell'isola di Lesbo (Grecia) (Coleoptera Tenebrionidae)
}

\begin{abstract}
Riassunto: Viene presa in esame la tenebrionidofauna dell' isola greca di Lesbo (Mar Egeo), sulla base di alcuni recenti reperti e dello studio critico della bibliografia nota. Attualmente sono 35 le specie di Tenebrionidi note per Lesbo, di cui le seguenti nove vengono qui segnalate per la prima volta: Erodius (Eodirosis) orientalis oblongus Solier, 1834, Helops (Helops) rossii Germar, 1817, Gonocephalum (Gonocephalum) costatum rugulosum (Küster, 1849), Dendarus (Pandarinus) moesiacus (Mulsant \& Rey, 1854), Uloma (Uloma) cypraea Kraatz, 1873, Trachyscelis aphodioides Latreille, 1809, Omophlus (Odontomophlus) pilosellus Kirsch, 1869, Megischia galbanata (Kiesenwetter, 1861) e Menephilus cylindricus cylindricus (Herbst, 1784). Dall'esame corologico si evidenzia una chiara impronta anatolica del popolamento di Tenebrionidi dell'isola.
\end{abstract}

Abstract: New records of tenebrionid beetles from Lesvos (Greece), with a summary of the fauna (Coleoptera Tenebrionidae). Published records of the 35 tenebrionid species occurring on the Greek island of Lesvos (Aegean Sea) are summarised and new ones are provided in detail. The following nine species are new to the island: Erodius (Eodirosis) orientalis oblongus Solier, 1834, Helops (Helops) rossii Germar, 1817, Gonocephalum (Gonocephalum) costatum rugulosum (Küster, 1849), Dendarus (Pandarinus) moesiacus (Mulsant \& Rey, 1854), Uloma (Uloma) cypraea Kraatz, 1873, Trachyscelis aphodioides Latreille, 1809, Omophlus (Odontomophlus) pilosellus Kirsch, 1869, Megischia galbanata (Kiesenwetter, 1861) and Menephilus cylindricus cylindricus (Herbst, 1784). A distributional analysis indicates that the tenebrionid beetle fauna of Lesvos is mainly composed of species also occurring in the Anatolian peninsula.

Key words: Tenebrionidae, Greece, Lesvos island, Chorology.

\section{INTRODUZIONE}

L'isola di Lesbo è situata nel Mar Egeo, al largo della penisola anatolica, con una distanza minima dal continente di circa $10 \mathrm{~km}$; ha una superficie di $1.630 \mathrm{~km}^{2}$ ed è per estensione la terza isola greca dopo Creta ed Eubea; l'isola si presenta prevalentemente collinosa e raggiunge la massima elevazione con il Monte Olimpo (968 m).

I Tenebrionidi dell'isola di Lesbo non sono mai stati oggetto di uno studio specifico; i reperti fino ad oggi noti sono quelli citati nei lavori di Sahlberg (1913), Schuster (1936), Koch (1948), Kühnelt (1965), Fattorini et al. (1999), Grimm \& Schawaller (2000), Fattorini (2002), Trichas (2008), Soldati \& Kakiopoulos (2010) e Soldati (2012); da notare che alcune delle specie citate da Sahlberg (1913) sono state omesse dal catalogo di Kühnelt (1965) e conseguentemente dai lavori successivi.

\section{MATERIALI E METODI}

Le specie di Tenebrionidi dell'isola di Lesbo sono qui elencate secondo l'ordine sistematico di Löbl et al. (2008); per ognuna vengono riportate le precedenti segnalazioni per l'isola (se presenti in letteratura), gli eventuali nuovi dati, la corologia ed eventuali osservazioni tassonomiche.

Nel presente lavoro vengono trattate anche le specie della tribù Lagriini e della sottofamiglia Alleculinae, considerato che la loro appartenenza alla famiglia Tenebrionidae appare ormai consolidata (Aalbu et al., 2002; Bouchard et al., 2005; Aalbu, 2006; Löbl et al., 2008; Bouchard et al., 2011).

I nuovi reperti sono stati raccolti da uno degli autori, Gianpaolo Ruzzante (GR), e dai colleghi Roberto Lisa (RL) e Zdeno Lucbauer (ZL). Questi esemplari sono conservati nelle collezioni private degli autori.

\section{TRATTAZIONE DELLE SPECIE}

Lagria (Lagria) hirta (Linnaeus, 1758) Precedenti SEgnalazioni. Sahlberg (1913: sub $L$. hirta L. e L. seminuda Reitt.).

NuOvi DATI. Mytilini, Kedro, 12-24.V.2015, 2 exx, RL leg.

\footnotetext{
*Piero Leo, Via Tola 21, 09128 Cagliari, Italia. E-mail: piero.leo@tiscali.it

**Gianpaolo Ruzzante, Via Giardini 132, 09127 Cagliari, Italia. E-mail: ruzzpa@tiscali.it
} 
DistribuZIOne. Specie a distribuzione sibirico-europea (Löbl et al., 2008).

OsSERVAZIONI. Lagria seminuda Reitter, 1889 (citata da Sahlberg, 1913) è attualmente considerata sinonimo di L. hirta (Löbl et al., 2008).

Erodius (Eodirosis) orientalis oblongus Solier, 1834 NuOvi DATI. Andissa, dune di Kambos, 23.III.2014, 6 exx, GR leg.; Sigri, dune di Faneromeni, 26.III.2014, 5 exx, GR leg.

DisTRIBUZIONE. La specie (s.1.) è distribuita dalla Grecia meridionale alla penisola anatolica; la ssp. oblongus è nota di alcune isole dell'Egeo (Fattorini et al., 1999) e della costa occidentale e meridionale dell'Anatolia (Kaszab, 1968; Keskin, 1999). Prima segnalazione per l'isola di Lesbo.

Pachyscelis (Parapachyscelis) villosa (Drapiez, 1820) PreCEDENTI SEGNALAZIONI. Sahlberg (1913); Schuster (1936); Kühnelt (1965).

NuOVI DATI. Agiasos, dintorni, 18.III.2014, 1 ex, GR leg.; Andissa, bivio per Eresos, 300 m, 25.III.2014, 5 exx, GR leg.; Andissa, foresta pietrificata, $250 \mathrm{~m}$, 26.III.2014, 3 exx, GR leg.; Anemotia, 16.III.2014, 2 exx, GR leg.; Eresos, Monastero Pithariou, 21.III.2014, 1 ex, GR leg.; Kalloni, dintorni, 23.III.2014, 2 exx, GR leg.; Loutropoli Thermis, Mt.Tavros, 12-24.V.2015, 4 exx, RL leg.; Mithimna, dintorni, 16.III.2014, 1 ex, GR leg.; Mytilini, castello, 12-24.V.2015, 11 exx, RL leg.; Nifida, 22.III.2014, 3 exx, GR leg.; Pedi, Mt. Kastelia, 12-24.V.2015, 1 ex, RL leg.; Skopelos, Mt. Petrovouni, 12-24.V.2015, 1 ex, RL leg.; Sigri, 26.III.2014, 2 exx, GR leg.; Sigri, castello, 12-24.V.2015, 1 ex, RL leg.; Vatera, strada per Ag. Focas, 25.III.2014, 3 exx, GR leg.

DistribuZIONE. Specie diffusa dalla Grecia al Medio Oriente (Löbl et al., 2008), particolarmente frequente nella penisola anatolica (Leo \& Fattorini, 2000) e presente in numerose isole dell'Egeo (Fattorini et al., 1999).

Pimelia (Camphonota) subglobosa (Pallas, 1781) s.l. PreCEDENTI Segnalazioni. Sahlberg (1913: sub P. polita var. euboica Boield., P. polita var. lesbicola var. nov., $P$. verruculifera Sol., $P$. verruculifera var. mongeneti Sol.); Schuster (1936: sub P. verruculifera Sol.). NuOvi Dati. Agiasos, Sanatorio, Mt. Keramos, 1224.V.2015, 1 ex, RL leg.; Agios Efstration, 140 m, 22.III.2014, 3 exx, GR leg.; Andissa, bivio per Eresos, 300 m, 25.III.2014, 3 exx, GR leg.; Andissa, foresta pietrificata, 250 m, 26.III.2014, 7 exx, GR leg.; Eresos, Monastero Pithariou, 21.III.2014, 2 exx, GR leg.; Filia, Mt.Kokines, 12-24.V.2015, 1 ex, RL leg.; Halinados, sito archeologico, 70 m, 19.III.2014, 9 exx, GR leg.; Kalloni, dintorni, 16.III.2014, 30 exx, GR leg.; NW Kalloni, Limonas Monastery, 24.III.2015, 5 exx, ZL leg.; Loutropoli Thermis, Mt.Tavros, 1224.V.2015, 2 exx, RL leg.; Mesa, sanctuary, 1224.V.2015, 2 exx, RL leg.; Mithimna, dintorni, 16.III.2014, 1 ex, GR leg.; Skopelos, Mt. Petrovouni, 12-24.V.2015, 2 exx, RL leg.; Tarti, 10 m, 24.III.2014, 5 exx, GR leg.

DistRIBUZIONE. La specie (s.l.) è presente dalla penisola balcanica fino agli Urali e al Kazakistan nord-occidentale (cfr. Leo \& Fattorini, 2000).

OsServazioni. I vari taxa di Pimelia del sottogenere Camphonota andrebbero revisionati sulla base di un ampio materiale; in questa sede riferiamo la variabilissima popolazione dell'isola di Lesbo a P. subglobosa, qui considerata monotipica (osservazioni e dati inediti di S. Fattorini \& P. Leo; cfr. anche Fattorini, 2002).

Dailognatha quadricollis carceli Solier, 1835

Precedenti Segnalazioni. Sahlberg (1913: sub D. carceli Sol. e D. quadricollis Brullé); Schuster (1936: sub D. caraboides v. caucasica Dbr.); Koch (1948).

NuOVI DATI. Achladeri, 18.III.2014, 1 ex, GR leg.; Andissa, foresta pietrificata, $250 \mathrm{~m}$, 26.III.2014, 1 ex, GR leg.; Anemotia, 16.III.2014, 1 ex, GR leg.; Apothikes, 26.III.2015, 2 exx, ZL leg.; Kalloni, dintorni, 16.III.2014, 1 ex, GR leg.; Loutropoli Thermis, Mt.Tavros, 12-24.V.2015, 2 exx, RL leg.; Mesa, sanctuary, 12-24.V.2015, 2 exx, RL leg.; Mytilini, castello, 1224.V.2015, 3 exx, RL leg.; Mithimna, dintorni, 16.III.2014, 1 ex, GR leg.; Pedi, Mt. Kastelia, 1224.V.2015, 1 ex, RL leg.; NE Tavari, 26.III.2015, 5 exx, ZL leg.; Vatera, strada per Ag. Focas, 25.III.2014, 1 ex, GR leg.

DistribuZIONE. Specie ampiamente diffusa (con più sottospecie delle quali sarebbe necessaria una revisione) nella penisola balcanica e nell'Anatolia, fino all'Armenia occidentale (Löbl et al., 2008); la ssp. carceli era già nota di Lesbo e di altre isole dell'Egeo (Fattorini et al., 1999).

Dichomma dardanum (Steven, 1829)

Precedenti Segnalazioni. Sahlberg (1913).

NuOvi DATI. Kambos, 25.III.2015, 1 ex, ZL leg.

DistribuZIone. Specie presente in Grecia e Anatolia 
(Löbl et al., 2008), nota di alcune isole dell'Egeo (Fattorini, 2002).

\section{Tentyria rotundata mittrei Solier, 1835}

PreCedenti Segnalazioni. Sahlberg (1913: sub T. angulata Brullè).

NuOvi DATI. Andissa, dune di Kambos, 23.III.2014, 9 exx, GR leg.; id., 25.III.2015, 2 exx, ZL leg.; Andissa, foresta pietrificata, $250 \mathrm{~m}, 26 . \mathrm{III} .2014,1 \mathrm{ex}$, GR leg.; Eresos, Monastero Pithariou, 21.III.2014, 13 exx, GR leg.; Mesa, sanctuary, 12-24.V.2015, 1 ex, RL leg.; Nifida, 22.III.2014, 1 ex, GR leg.; Sigri, dune di Faneromeni, 26.III.2014, 11 exx, GR leg.; Skala Kalloni, 21.III.2014, 13 exx, GR leg.

DistRiBUZIONE. La specie (s.l.) è diffusa dalla penisola balcanica all'Iran (Löbl et al., 2008); la ssp. mittrei è ampiamente distribuita in Anatolia ed è presente in alcune isole egee (Fattorini et al., 1999).

Zophosis (Oculosis) punctata punctata Brullé, 1832 PreCedenti Segnalazioni. Sahlberg (1913: sub $Z$. punctata Brullé, Z. punctata v. kollari Deyr., Z. truquii Deyr.); Schuster (1936: sub Z. punctata a. vescoi Deyr.); Kühnelt (1965).

NuOvi DATI. Andissa, dune di Kambos, 23.III.2014, 1 ex, GR leg.; Eresos, Monastero Pithariou, 21.III.2014, 2 exx, GR leg.; Filia, Mt.Kokines, 12-24.V.2015, 1 ex, RL leg.; Sigri, castello, 12-24.V.2015, 1 ex, RL leg. DisTRIBUZIONE. Specie a distribuzione turano-mediterranea (Löbl et al., 2008), nota di numerose isole dell'Egeo (Fattorini, 2002).

\section{Helops (Helops) rossii Germar, 1817}

NuOVI DATI. Agiasos, dintorni, 18.III.2014, 1 resto, GR leg.

DistribuZIONE. Specie diffusa nell'Europa meridionale e penisola anatolica (Löbl et al., 2008); per le isole dell'Egeo era nota solo di Creta e Thasos (Fattorini et al., 1999; Fattorini \& Fowles, 2005). Prima segnalazione per l'isola di Lesbo.

Probaticus (Pelorinus) peslieri Soldati, 2012

Precedenti Segnalazioni. Sahlberg (1913: sub Helops corrugosus Seidl.); Soldati (2012).

Nuovi Dati. SE Agios Paraskevi, 24.III.2015, 10 exx, ZL leg.; Mesa, sanctuary, 12-24.V.2015, 1 ex, RL leg.; 3 km NW Agra, 500 m, 25.III.2015, 9 exx, ZL leg.

DistribuZione. Specie endemica dell'isola di Lesbo
(Soldati, 2012). Nota sinora sulla sola serie tipica

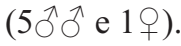

OSSERVAZIONI. Riteniamo verosimile che la citazione di Sahlberg (1913), relativa a Probaticus (Pelorinus) corrugosus (Seidlitz, 1896), sia da riferire a $P$. peslieri; P. corrugosus è una specie enigmatica, descritta di "Klein-Asien" (Anatolia) e "Astrabad" (oggi Gorgan, capoluogo della regione iraniana del Golestan) e a quanto ci risulta non più raccolta dopo la descrizione; da notare che Löbl et al. (2008) indicano P. corrugosus solo per Turchia asiatica e Grecia (quest'ultima presumibilmente proprio in base alla citazione di Sahlberg per Lesbo).

Nel descrivere $P$. peslieri, Soldati (2012) ha rimarcato come peculiare un carattere dell'unica femmina della serie tipica: "the $3^{\text {rd }}$ interstria with a strong costa on the disc of elytra, a spectacular and unique morphological character into the genus Probaticus"; questo carattere è invece assente in tutti gli undici esemplari di sesso femminile da noi esaminati (Fig. 1) e rite-

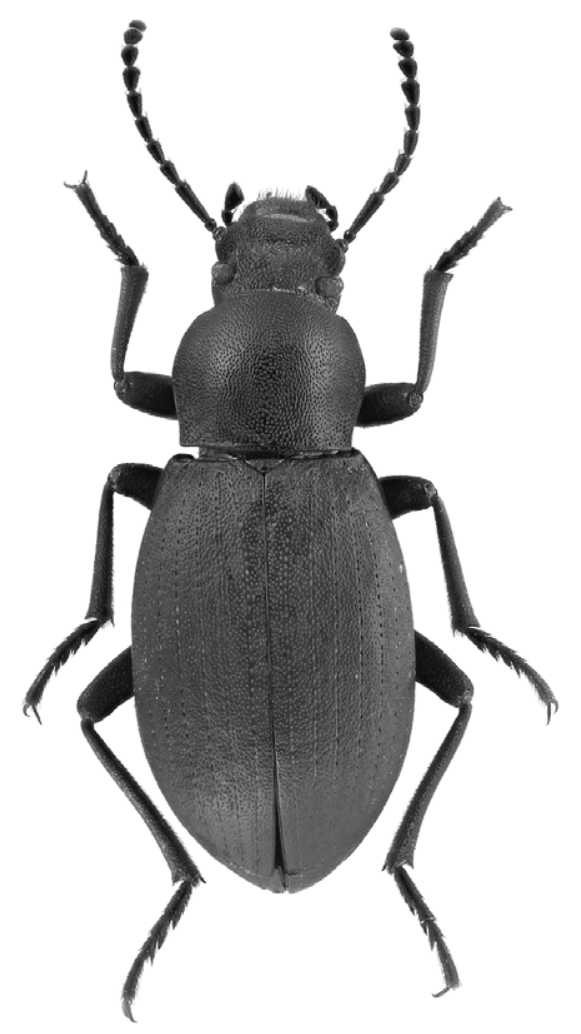

Fig. 1. Probaticus (Pelorinus) peslieri Soldati, 2012 (Isola di Lesbo, 3 km NW Agra, 500 m, 25.III.2015, Z. Lucbauer leg.): habitus + , lunghezza $14 \mathrm{~mm}$ (foto D. Sechi). 
niamo quindi che il paratipo femmina sia un esemplare aberrante (cfr. Soldati, 2012: Fig. 2).

Raiboscelis coelestinus coelestinus (Waltl, 1838) Precedenti Segnalazioni. Schuster (1936: sub Helops coelestinus v. quadraticollis Küst.); Kühnelt (1965: sub R. coelestinus Waltl, 1838 e R. coelestinus a. quadraticollis Küst., 1850]).

Distribuzione. Specie diffusa in Anatolia e presente in alcune isole dell'Egeo (Fattorini et al., 1999).

\section{Gonocephalum (Gonocephalum) costatum rugulo-} sum (Küster, 1849)

NuOVI DATI. 3 km NW Agra, 500 m, 25.III.2015, 1 ex, ZL leg.; Mytilini, castello, 12-24.V.2015, 1 ex, RL leg. DistribuZione. Specie diffusa nel Mediterraneo orientale; la sottospecie rugulosum, descritta di Anatolia, è nota anche di Armenia, Cipro, Iran, Israele e Siria (Löbl et al., 2008). Prima segnalazione per l'isola di Lesbo.

Gonocephalum (Gonocephalum) rusticum (A.G. Olivier, 1811)

Precedenti Segnalazioni. Schuster (1836); Kühnelt (1965).

DistRIBUZIONE. Specie ad ampia distribuzione nella regione paleartica (Löbl et al., 2008), presente in varie isole dell'Egeo (Fattorini et al., 1999).

Opatroides punctulatus Brullé, 1832

PRECEDENTI SEGNALAZIONI. Kühnelt (1965).

NuOvi DATI. Achladeri, 18.III.2014, 10 exx, GR leg.; E Agios Paraskevi, 29.III.2015, 1 ex, ZL leg.; Anemotia, 16.III.2014, 7 exx, GR leg.; Apothikes, 26.III.2015, 1 ex, ZL leg.; Eresos, Monastero Pithariou, 21.III.2014, 2 exx, GR leg.; Filia, Mt.Kokines, 12-24.V.2015, 16 exx, RL leg.; Halinados, sito archeologico, 70 m, 19.III.2014, 3 exx, GR leg.; Kalloni, dintorni, 16.III.2014, 11 exx, GR leg.; Loutropoli Thermis, Mt.Tavros, 12-24.V.2015, 14 exx, RL leg.; Mithimna, dintorni, 16.III.2014, 6 exx, GR leg.; Pedi, Mt. Kastelia, 12-24.V.2015, 1 ex, RL leg.; Skopelos, Mt. Petrovouni, 12-24.V.2015, 1 ex, RL leg.; NE Tavari, 26.III.2015, 7 exx, ZL leg.; Vatera, strada per Ag. Focas, 25.III.2014, 6 exx, GR leg.

DistRibuZIONE. Specie ad ampia distribuzione nella regione paleartica e nella regione subsahariana (Löbl et al., 2008); è nota di numerose isole dell'Egeo (Fattorini, 2002).
Opatrum (Opatrum) geminatum Brullé, 1832

PRECEDENTI SEGNALAZIONI. Sahlberg (1913).

Distribuzione. Specie del Mediterraneo orientale (Löbl et al., 2008), citata per le isole dell'Egeo unicamente di Lesbo.

Opatrum (Opatrum) obesum A.G. Olivier, 1811 Precedenti Segnalazioni. Sahlberg (1913). NuOvi DATI. Kambos, 25.III.2015, 1 ex, ZL leg.; SE Agios Paraskevi, 24.III.2015, 1 ex, ZL leg.

DistriBUZIONE. Specie nota di Italia meridionale, Sicilia, Grecia, Turchia e Siria (Leo \& Fattorini, 2002), citata anche di Iraq da Löbl et al. (2008), dato quest'ultimo che meriterebbe di essere confermato; la specie è segnalata di varie isole dell'Egeo (Fattorini, 2002).

OsSERVAZIONI. Fattorini \& Fowles (2005) riportano per Lesbo anche la specie Opatrum sabulosum (Linné, 1758); trattasi di un refuso, confermato anche da uno degli autori (Fattorini: comunicazione personale): questa specie, citata dell'isola di Lemnos da Kühnelt (1965: sub Opatrum sabulosum lucifugum Küster, 1849), sembra assente a Lesbo.

Dendarus (Pandarinus) messenius (Brullé, 1832) Precedenti Segnalazioni. Trichas (2008).

DistribuZIONE. Specie a distribuzione balcanico-anatolica (Löbl et al., 2008), già nota di alcune isole dell'Egeo (Fattorini et al., 1999; Trichas, 2008)

Dendarus (Pandarinus) moesiacus (Mulsant \& Rey, 1854)

NuOVI DATI. Kambos, 25.III.2015, 1 ex, ZL leg.; Mithimna, dintorni, 16.III.2014, 1 resto, GR leg.; Sigri, 26.III.2014, 1 ex, GR leg.

DisTRIBUZIONE. Specie a distribuzione balcanico-anatolica (Löbl et al., 2008), già nota di alcune isole dell'Egeo (Fattorini et al., 1999; Trichas, 2008). Prima segnalazione per l'isola di Lesbo.

Micrositus orbicularis Mulsant \& Rey, 1854

PreCEDEnTI SEgnalazioni. Sahlberg (1913).

Distribuzione. Specie nota di Lesbo, Creta e di alcune delle isole Cicladi (Fattorini et al. 1999); secondo Scupola \& Lo Cascio (2001) la presenza a Lesbo sarebbe meritevole di conferma.

Colpotus sulcatus (Ménétriés, 1838)

Precedenti segnalazioni. Grimm \& Schawaller (2000). 
DistribuZIONE. Specie presente in Anatolia e in alcune isole dell'Egeo (Grimm \& Schawaller, 2000)

Pedinus (Pedinus) quadratus Brullé, 1832

PreCEDEnTI SEGNALAZIONI. Sahlberg (1913); Kühnelt (1965).

DistribuZIONE. Specie a distribuzione balcanica, nota di varie isole dell'Egeo (Fattorini, 2002)

\section{Cephalostenus orbicollis (Ménétriés, 1836)}

Precedenti Segnalazioni. Sahlberg (1913); Koch (1948).

NuOVI DATI. Mytilini, castello, 12-24.V.2015, 2 exx, RL leg.

DistribuZIONE. Specie del Mediterraneo orientale (Löbl et al., 2008) la cui presenza nella Grecia continentale è stata confermata solo di recente (Liberto \& Leo, 2006); è presente in varie isole dell'Egeo (Fattorini, 2002).

Uloma (Uloma) cypraea Kraatz, 1873

NuOvi DATI. Agiasos, dintorni, 18.III.2014, 1 ex, GR leg.

Distribuzione. Specie nota di Grecia, Anatolia, Cipro e Siria; per la Grecia si conosceva solo una segnalazione recente dell'isola Eubea (Leo \& Fattorini, 2002). Prima segnalazione per l'isola di Lesbo.

Platydema europaea Laporte \& Brullé, 1831

Precedenti Segnalazioni. Sahlberg (1913).

Distribuzione. Specie a distribuzione prevalentemente mediterranea (Löbl et al., 2008); per le isole dell'Egeo è nota di Lesbo e di Thasos (Fattorini \& Fowles, 2005).

\section{Corticeus (Corticeus) pini (Panzer, 1799)}

PreCEDENTI SEGNALAZIONI. Sahlberg (1913: sub Hypophloeus pini Panz.).

Distribuzione. Specie ad ampia distribuzione nella regione paleartica (Löbl et al., 2008), per le isole dell'Egeo è nota esclusivamente di Lesbo e di Siros (Kühnelt, 1965).

Crypticus (Platycrypticus) brusteli Soldati \& Kakiopoulos, 2010

Precedenti Segnalazioni. Soldati \& Kakiopoulos (2010).

DistribuZione. Specie endemica dell'isola di Lesbo (Soldati \& Kakiopoulos, 2010).
Crypticus (Platycrypticus) castaneus Baudi di Selve, 1876

Precedenti Segnalazioni. Soldati \& Kakiopoulos (2010).

DistribuZIone. Specie nota dell'Anatolia e di alcune isole dell'Egeo (Soldati \& Kakiopoulos, 2010).

Phaleria (Phaleria) bimaculata (Linnaeus, 1767) Precedenti segnalazioni. Sahlberg (1913: sub P. bimaculata L. e P. bimaculata var. nigricordis $\mathrm{O}$. Schn.).

DistribuZIONE. Specie ad ampia distribuzione mediterranea (Löbl et al., 2008), presente in varie isole dell'Egeo (Fattorini et al., 1999).

Trachyscelis aphodioides Latreille, 1809

NuOvi DATI. Andissa, dune di Kambos, 23.III.2014, 3 exx, GR leg.

DisTRIBUZIONE. Specie ad ampia distribuzione prevalentemente mediterranea (Löbl et al., 2008), già nota di alcune isole dell'Egeo (Fattorini, 2002). Prima segnalazione per l'isola di Lesbo.

Hymenalia (Hymenalia) gravida (Küster, 1850) PRECEDENTI SEGNALAZIONI. Sahlberg (1913).

DisTRIBUZIONE. Specie a distribuzione balcanico-anatolica (Löbl et al., 2008); non ci risultano altre segnalazioni per le isole dell'Egeo.

Megischia galbanata (Kiesenwetter 1861)

NuOvi DaTI. Mytilini, Kedro, 12-24.V.2015, 1 ex, RL leg.

DisTRIBUZIONE. Specie a distribuzione balcanico-anatolica (Löbl et al., 2008); non ci risultano altre segnalazioni per le isole dell'Egeo. Prima segnalazione per l'isola di Lesbo.

Omophlus (Odontomophlus) flavipennis Küster, 1849

Precedenti Segnalazioni. Sahlberg (1913).

NuOVI DATI. Agiasos, Sanatorio, Mt. Keramos, 1224.V.2015, 1 ex, RL leg.; Kalloni, Moni Limonos, 1224.V.2015, 1 ex, RL leg.

Distribuzione. Specie dell'Europa orientale e Asia occidentale (Löbl et al., 2008); non ci risultano altre segnalazioni per le isole dell'Egeo.

Omophlus (Odontomophlus) pilosellus Kirsch, 1869 NuOvi DATI. Agiasos, Sanatorio, Mt. Keramos, 12- 
24.V.2015, 2 exx, RL leg.; Kalloni, Moni Limonos, 12-24.V.2015, 5 exx, RL leg.

Distribuzione. Specie nota di Croazia, Grecia, Anatolia e Iraq (Löbl et al., 2008). Prima segnalazione per l'isola di Lesbo.

Podonta frater Seidlitz, 1896

Precedenti SEGNALAZIONI. Sahlberg (1913).

Distribuzione. Specie nota dell'Anatolia (Löbl et al., 2008); non ci risultano altre segnalazioni per le isole dell'Egeo.

Menephilus cylindricus cylindricus (Herbst, 1784) NuOVI DATI. Agiasos, dintorni, 18.III.2014, 1 ex, GR leg.; SE Agios Paraskevi, 24.III.2015, 3 exx, ZL leg. DisTRIBUZIONE. Specie ad ampia distribuzione nella regione paleartica occidentale (Löbl et al., 2008), già nota di alcune isole dell'Egeo (Fattorini, 2002). Prima segnalazione per l'isola di Lesbo.

\section{CONCLUSIONI}

In base ai dati attualmente disponibili, ricavati sia dall'analisi critica della letteratura e sia dai nuovi recenti reperti, la tenebrionidofauna di Lesbo consta di 35 specie note (29 non considerando i Lagriini e gli Alleculinae), di cui 9 nuove per l'isola. Benché le attuali conoscenze sul popolamento dei Tenebrionidi dell'area egea non siano del tutto esaustive, da un confronto con quanto noto per le altre isole osserviamo che il numero totale di taxa presenti a Lesbo appare inferiore solo a quello di Creta, Eubea, Rodi e Naxos (cfr. Fattorini, 2002).

Dal punto di vista corologico, è possibile suddividere i Tenebrionidi di Lesbo nelle seguenti categorie già utilizzate da Fattorini et al. (1999) e Fattorini (2002):

- taxa a distribuzione più o meno ampia, ma comunque presenti sia nella penisola balcanica che in
Anatolia (23 specie): Lagria hirta, Pachyscelis villosa, Pimelia subglobosa, Dailognatha quadricollis carceli, Dichomma dardanum, Zophosis punctata punctata, Helops rossii, Gonocephalum rusticum, Opatroides punctulatus, Opatrum geminatum, O. obesum, Dendarus messenius, D. moesiacus, Cephalostenus orbicollis, Platydema europaea, Corticeus pini, Phaleria bimaculata, Trachyscelis aphodioides, Hymenalia gravida, Megischia galbanata, Omophlus flavipennis, Omophlus pilosellus, Menephilus cylindricus cylindricus.

- taxa presenti in Anatolia ma assenti nella penisola balcanica (8 specie): Erodius orientalis oblongus, Tentyria rotundata mittrei, Raiboscelis coelestinus coelestinus, Gonocephalum costatum rugulosum, Colpotus sulcatus, Uloma cypraea, Crypticus castaneus, Podonta frater.

- taxa presenti nella penisola balcanica ma assenti in Anatolia (1 specie): Pedinus quadratus.

- taxa presenti in più di un'isola dell'Egeo e assenti sia in Anatolia che nella penisola balcanica (1 specie): Micrositus orbicularis.

- taxa endemici (2 specie): Probaticus peslieri, Crypticus brusteli.

Dall'analisi dei dati sopra riportati si evince un'origine prevalentemente anatolica del popolamento di Tenebrionidi di Lesbo, chiaramente attribuibile alla posizione geografica dell'isola, situata in prossimità della costa turco-asiatica.

\section{RINGRAZIAMENTI}

Si ringraziano per la collaborazione i seguenti amici e colleghi: Simone Fattorini (Roma) per gli utili consigli e l'aiuto nella ricerca bibliografica; Roberto Lisa (Firenze) e Zdeno Lucbauer (Kettering) per i reperti forniti; Daniele Sechi (Cagliari) per la fotografia di Probaticus peslieri.

\section{BIBLIOGRAFIA}

AALBU R.L., 2006 - Where are we at: assessing the currents state of Tenebrionidae systematics on a global scale (Coleoptera: Tenebrionidae). Cahiers Scientifiques. Centre de Conservation et d'Étude des Collections (Lyon), 10: 55-70.

Aalbu R.L., Triplehorn C.A., Campbell J.M., Brown K.W., Somerby R.E., Thomas D.B., 2002 - Family 106. Tenebrionidae Latreille 1802 (pp. 463-509). In: Arnett R.H.J., Thomas M.C., Skelley P.E. \& Frank J.H. (eds.), American beetles. Volume 2. Polyphaga: Scarabaeoidea through Curculionoidea. CRC Press, Boca Raton, 861 pp.

Bouchard P., Lawrence J.F., Davies A.E., Newton A.F., 2005 - Synoptic classification of the world Tenebrionidae (Insecta: Coleoptera) with a review of family-group names. Annales Zoologici, 55: 499-530. 
Bouchard P., Bousquet Y., Davies A.E., Alonso-Zarazaga M.A., Lawrence J.F., Lyal C.H.C., Newton A.F., Reid C.A.M., Schmitt M., SLIPINSKi S.A., Smith A.B.T., 2011 - Family-group names in Coleoptera (Insecta). ZooKeys, 88: 1-972.

FATTORINI S., 2002 - Biogeography of the tenebrionid beetles (Coleoptera, Tenebrionidae) on the Aegean Islands (Greece). Journal of Biogeography, 29: 49-67.

FATtorini S., Fowles A., 2005 - A biogeographical analysis of the tenebrionid beetles (Coleoptera, Tenebrionidae) of the island of Thasos in the context of the Aegean Islands (Greece). Journal of Natural History, 39(46): 3919-3949.

Fattorini S., Leo P., SAlvati L., 1999 - Biogeographical observations on the Darkling beetles of the Aegean Islands (Coleoptera, Tenebrionidae). Fragmenta Entomologica, 31: 339-375.

Grimm R., Schawaller W., 2000 - The genus Colpotus Mulsant \& Rey (Coleoptera: Tenebrionidae) in the Eastern Mediterranean Region, with descriptions of two new species. Stuttgarter Beiträge zur Naturkunde, Serie A (Biologie), 615: 1-15.

Kaszab Z., 1968 - Ergebnisse zoologischer Sammelreisen in der Türkey. Coleoptera Tenebrionidae. Annalen des Naturhistorischen Museums in Wien, 72: 451-463.

KESKIN B., 1999 - Balçova Baraji (İzmir; Türkiye) civari Tenebrionidae (Coleoptera) faunasi. Türkiye entomoloji derneği, 23(3): 221-224.

Косн C., 1948 - Die Tenebrioniden Kretas (Col.). Mitteilungen der Münchener Entomologischen Gesellschaft, 34(1944): 255363.

KÜHnelt W., 1965 - Tenebrionidae. In: Kanellis A. (ed.), Catalogus Faunae Graeciae. To Vouno: 1-60.

Kwieton E., 1982 - Contributions ultérieures à la connaissance du genre Pimelia F. (Col., Tenebrionidae). Annotationes Zoologicae et Botanicae, 145: 1-38.

Leo P., FAtTorini S., 2000 - The zoogeographical composition and distribution of the Anatolian Pimeliini (Coleoptera, Tenebrionidae). Biogeographia, 21: 399-427.

Leo P., FAtToRini S., 2002 - New records of tenebrionid beetles from the Aegean Islands (Coleoptera, Tenebrionidae). Biocosme Mèsogèen, 18(4): 157-165.

Liberto A., Leo P., 2006 - Una nuova Halammobia del Peloponneso e nuovi dati faunistici sui Tenebrionidi della Grecia (Col. Tenebrionidae). Fragmenta Entomologica, 38(2): 251-277.

Löbl I., Ando K., Bouchard P., Iwan D., Lillig M., Masumoto K., Merkl O., Nabozhenko M., Novák V., Petterson R., Schawaller W., Soldati F., 2008 - Family Tenebrionidae Latreille, 1802 (pp. 105-325). In: Löbl I., Smetana A. (eds.), Catalogue of Palaearctic Coleoptera. Volume 5. Tenebrionoidea. Apollo Books, Stenstrup, 670 pp.

SAhlBerg J.R., 1913 - Coleoptera mediterranea orientalis quae in Aegypto, Palestina, Syria, Caramania collegerunt John Sahlberg et Unio Saalas. Öfversigt af Finska Vetenskaps-Societetens Förhandlingar, 55(1912-1913): 1-282.

Schuster A., 1936 - Die Tenebrioniden (Col.)-Ausbeute von Prof. Dr. Franz Werner und Kustos Dr. Otto Wettstein von den Griechischen, Agâischen Inseln und dem italienischen Dodekanes in den Jahren 1934 und 1935. Sitzungsberichten der Akademie der Wissenschaften in Wien, Mathematisch-naturwissenschaftliche Klasse, Abteilung I, 145: 65-80.

Scupola A., Lo Cascio P., 2001 - Nouvelle données sur les Tenebrionidae des îles Pano Koufonissi et Folegandros (Cyclades, Grèece) (Coleoptera). Biocosme Mésogéen, 17: 209-217.

Soldati F., 2012 - A new species of the genus Probaticus Seidlitz, 1896 from Greece (Insecta: Coleoptera: Tenebrionidae). Annales Zoologici, 62(2): 221-225.

Soldati S., KaKiopoulos G., 2010 - A review of the genus Crypticus Latreille, 1817 in Greece with description of a new species (Insecta: Coleoptera: Tenebrionidae). Annales Zoologici, 60(2): 225-230.

Trichas A., 2008 - The genus Dendarus Latreille, 1829 (Coleoptera, Tenebrionidae: Dendarini) in Greece (A systematic account of the genus with description of a new species and four new systematic combinations). In: Makarov S.E., Dimitrijević R.N. (eds.), Advances in Arachnology and Developmental Biology. Inst. Zool. Belgrade, BAS Sofia, Fac. Life Sci. Vienna, SASA Belgrade \& UNESCO MAB Serbia. Vienna - Belgrade - Sofia, Monographs, 12: 417-462. 